\title{
Design Factors Extraction and Application of Five Dynasties Yaozhou Porcelain Decoration
}

\author{
Qin Song ${ }^{1, \mathrm{a}}$, Wang $\mathrm{Yi}^{1}$ \\ ${ }^{1}$ Xi'an University of Technology, School of Art and Design, 710054, Xi'an, China
}

\begin{abstract}
For the better development of Yaozhou Kiln, the article is proposed a research method about cultural and creative products based on the example of extracting the lotus patterns on the Yanzhou Kiln in the Five Dynasties. By collecting data and extracting forms, through the combination of qualitative and quantitative, it mines the obscure information of the Yaozhou porcelain ornamentation in the Five Dynasties. The article analyzes the form evaluations of the 18 representative lotus patterns in the Five Dynasties by semantic difference method, extracts their stylized inheritance and recessive genes and makes the conclusion to guide the cultural and creative products design of the Yaozhou Kiln, which not only supplies a fresh design perspective for the innovation development of the famous domestic traditional kilns as a reference, but also a useful supplements for the research of the design art methodology.
\end{abstract}

\section{Introduction}

The core area of Yaozhou Kiln is located in Tongchuan City, Shaanxi Province. Yaozhou Porcelain is a representative of the northern celadon with dignified and elegant styling, bright colors, distinctive patterns, beautiful and orderly structure, novel and unique decoration techniques. The ceramic decorative pattern is an important part of the Yaozhou Kiln ceramics. It not only records and expresses the unique lifestyle and aesthetic concepts of the people at that time, but also reflects the intelligence and wisdom of the Yaozhou people in the creation of the ornament based on the specific cultural environment. Due to various factors such as geographical environment and commercial information, Yaozhou Kiln Ceramics gradually lost its glory in its period of great prosperity. Its inheritance, development and branding are also facing much difficulties. Therefore, it is proposed that a research method about extracting the design factors of the emblazonry on Yaozhou Kiln ceramics in the Five Dynasties.

At present, domestic methods and applications for the refining research of cultural products are still at an initial stage. Most of the existing researches include the refining of architectural culture factors, cultural relics form factors, and dress pattern factors. Yan Bingyi et al. ${ }^{[1]}$ took painted pottery culture of Banpo relics as the research object, introduced the design process based on genetic engineering, and constructed the style cultural gene pool; Wang Weiwei et al. ${ }^{[2]}$ adopted Salt culture and the costume culture of Han Dynasty as the research object, and he took eye movement experiments and spectrogram analysis methods to extract cultural factors and apply them to product design; Zhou Xiaozhou et al. ${ }^{[3]}$ used FSA method and Delphi method to statistically summarize dominant DNA genetic factors of product family from the microscopic perspective, and applied the SD method to find recessive DNA genetic factors from a macro perspective, which provides feasibility guidance for product family design; Jay PM ${ }^{[4]}$ proposed a design language extraction and evolution algorithm based on cultural algorithms, using Buick as an example for application verification; Raphaelle ${ }^{[5]}$ et al. studied the recognition method that integrates customer requirements into the semantics of product initial design, and studied the relationship between design and semantics through regression analysis.

The above research used different methods to extract cultural product factors for different subjects and provided effective solutions to the problem. However, the research on the use of the ceramic design factors of a specific period of time has rarely been carried out. In this paper, the design method combines qualitative and quantitative analysis, and based on an in-depth analysis of the existing methods of researching design factors, a set of method models based on correlation analysis and in line with the design language extraction and derivation design are proposed to enable traditional culture and products design practices are better integrated with each other.

\section{Construction of research process}

Yaozhou porcelains in different time have its unique cultural connotations and artistic expressions in different periods. As a special art form that integrates practicality and art, the cultural and artistic information it contains is rich and varied. Yaozhou Porcelain is also the convergence product of multiculturalism and contains many cultural connotations. Therefore, this article mainly selects Five Dynasties of Yaozhou Porcelain

\footnotetext{
* Corresponding author: ${ }^{\mathrm{a}}$ qinbinbin@126.com
} 
ornamentation as the research object. Based on a deep understanding of the typical art features and craftsmanship culture of Yaozhou Kiln celadon of the Five Dynasties, a large number of sample data were collected and selected through shooting and scanning, and the selected sample pictures were processed and simplified to form the sample curve using flat software. After samples is collected and processed, the perceptual vocabulary is screened by methods such as clustering and fuzzy comprehensive evaluation. The key perceptive adjectives are selected through questionnaire survey, and the representative lotus patterns of the Yaozhou porcelains of the Five Dynasties are selected as Samples of patterns are analyzed based on the semantic difference method to analyze statistical representative samples, then reference thresholds and other design criteria are obtained. Finally, computer-aided design is used to verify whether the scheme of resulting diagram meets design demands.

\section{Factor extraction}

\subsection{Method introduction}

This study is divided into two steps. Firstly, the clustering method is used to cluster the samples, the most suitable group is divided, and the eigenvalues of each group are identified. And then, the semantic difference method is applied to analyze and evaluate a representative sample with the semantic difference components table. The design-related evaluation data was obtained ${ }^{[6]}$.

The advantage of clustering method is that it does not add too much judgment basis, but identifies the hidden objective laws among the data, and then recognizes and clusters them, so that the clustering result is more objective and reliable ${ }^{[7]}$. The specific process is as follows: (1) Describe the characteristics of objective traits visible in the ceramic decoration of Yaozhou Kiln, including the composition of a picture, main body, structure, lines, arrangement ways etc. (2) Correspond one by one with the characteristics of ceramic decoration in Yaozhou Kiln.

\subsection{Participants selection}

There is a total of 42 participants, of whom 5 are experts, 3 are artisans, 3 are workers working in Yaozhou Kiln, 2 are sales staff of Yaozhou Kiln Ceramics, 4 are product designers, 12 are design students at school, and 13 people are Yaozhou Kiln Ceramic users. Participants including 23 females and 19 males, who aged 20 to 45 years old. All of them have a certain understanding of Yaozhou Kiln.

\subsection{Building Sensitive Vocabulary Database}

First, a perceptual vocabulary database was established based on the experimental object design. According to the previous study, the factors affecting the decorative features were divided into composition, theme, structure, and lines in a sequence according to the proportion of importance. Through consulting and discussing with relevant experts and analyzing and researching a large amount of ceramic decoration materials of the Yaozhou Kiln during the period of the Five Dynasties, 40 style inheritance features affecting ornamentation were listed ${ }^{[8]}$. Select relevant vocabulary from them and remove adjectives that are not related to the experimental goal or have similar meanings. According to Osgood's research results, we selected 12 pairs of adjective pairs from emotional factors, mode factors and structural factors. Each of these factors had 4 groups, as shown in the Table1.

Table.1 Image adjective pairs.

\begin{tabular}{llll}
\hline Types & Emotional factors & Mode factors & Structural factors \\
\hline 1 & Untrammeled - Exquisite & Plump - Thin & Delicate - Vigorous \\
2 & Intensity - Preciseness & Elegance - Austere & Concise - Complex \\
3 & Swank - Connotative & Gorgeous - Fresh & Vivid - Natural \\
4 & Lively - Sober & Robust - Flexible & Smooth - Rigid \\
\hline
\end{tabular}

\subsection{Data collection, statistics and analysis}

The emblazonry is a part of the body of the ceramics, which has aesthetic and ornamental decorative features. For future generations, it is also an important historical research material that studies the customs, aesthetics, culture, and major historical events at that time. In the history of Yaozhou Kiln, it has extensively absorbed multicultural factors, including religious subjects, making its pattern rich and varied, and its expressiveness is gorgeous. The Yaozhou Kiln in the contains a large number of animal and plant patterns of Buddhist cultural factors. The lotus pattern is the most representative because ancient people often use the cover and the lotus petals to decorate artifacts. The lotus pattern is also an early pattern used by Yaozhou Kiln. In the Five Dynasties, the Tang and early Song dynasties, porcelains and tricolors of various glazes were adorned with plastic and engraved upward and covered lotus petals ${ }^{[9]}$.

The lotus pattern is one of the most typical patterns of the Yaozhou Kiln celadon of the Five Dynasties. Five, six, and seven petals are the most common patterns. There are 12 or more patterns, and the lotus petals are fat and mellow, and the patterns are regular. It continued to be used in the early Song Dynasty, but its shape became thinner and less visible later. This article first collected the pattern of lotus patterns in the Five Dynasties period. The lotus patterns were divided into 18 patterns numbered P1-P18. The analysis of artistic features and intentional symbology was conducted separately. The samples were analyzed using the semantic difference method. Then the inheritance relationship of the pattern elements was extracted and the pattern gene was extracted to prepare for the next design study. As shown in Figure 1. 


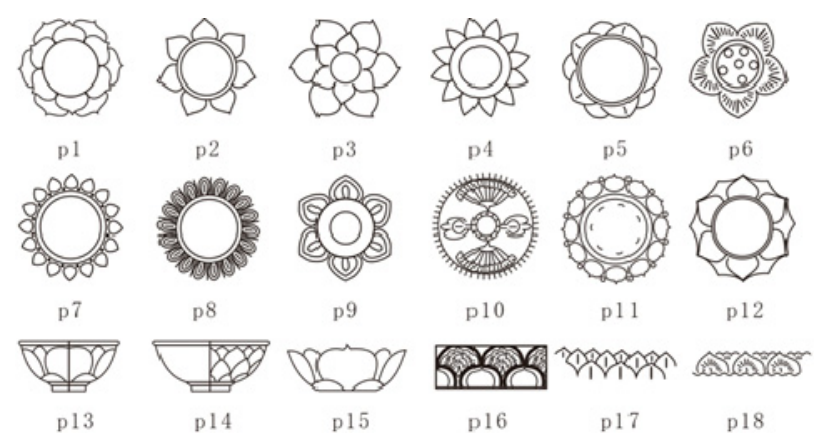

Figure 1. Samples of the lotus decoration of the Yaozhou Kiln in the Five Dynasty.

P1. Huangbao Kiln Site P2. Huangbao Kiln Site P3. Huangbao Kiln Site P4. Pingquan Xiaojigou Liao Mausoleum P5. Dingzhou Jingzhi Temple Taji P6. Faku Museum Ye Maotai M22 P7. Yaozhou Kiln Museum Tibet P8. Shaanxi History Museum P9. Yaozhou Kiln Museum Museum P10. Shaanxi History Museum Collection P11. Lotus petals (bed golden groove M5 lotus care) P12. Huangbao Kiln site P13. Huangbao Kiln Site A type IV type lotus P14. Faku Ye Maotai M7 P15. Lotus petal pattern (Yao Maotai M7 bowl) P16. Lotus petal pattern (Ye-Mai Taiwan M7 bowl) P17. Huangbao Kiln Site seal lotus petals P18. Huangbao Kiln Site paste model

A questionnaire survey was conducted based on the semantic difference method to understand the target group's emotional evaluation of the product. The target group can score at the corresponding position in each perceptual adjective based on its perception of each product. Finally, statistical analysis of the questionnaire survey data provides the basis for subsequent development. In this round, the expert scoring method was used. A total of 12 industry experts and designers were selected to conduct the questionnaire survey. Figure 3 shows an example of a sample questionnaire.

\begin{tabular}{|c|c|c|c|c|c|c|c|c|}
\hline & -3 & -2 & -1 & 0 & 1 & 2 & 3 & \\
\hline Untrammeled & & & & & & & & Exquisite \\
\hline Plump & & & & & & & & Thin \\
\hline Delicate & & & & & & & & Vigorous \\
\hline Intensity & & & & & & & & Preciseness \\
\hline Elegance & & & & & & & & Austere \\
\hline Concise & & & & & & & & Complex \\
\hline Swank & & & & & & & & Connotative \\
\hline Gorgeous & & & & & & & & Fresh \\
\hline Vivid & & & & & & & & Natural \\
\hline Lively & & & & & & & & Sober \\
\hline Robust & & & & & & & & Flexible \\
\hline Smooth & & & & & & & & Rigid \\
\hline
\end{tabular}

Figure 3. Example of a Five Dynasties Yaozhou porcelain ornament questionnaire.

The weighted average method is used to count the expert scores of different adjectives in each sample, and the calculation formula according to the weighted average value is formula (1):

$$
x=\frac{1}{n} \sum_{k=1}^{n} x_{k} f_{k}
$$

$\mathrm{x}$ - the score given by the expert;

$\mathrm{f}$ - the number of occurrences of each score;

$\mathrm{n}-$ the number of experts.
From formula (1), The weighted average number of adjectives in each sample was calculated by $\sum_{k=1}^{n} f_{k}=n$, and the results are shown in Figure 2.

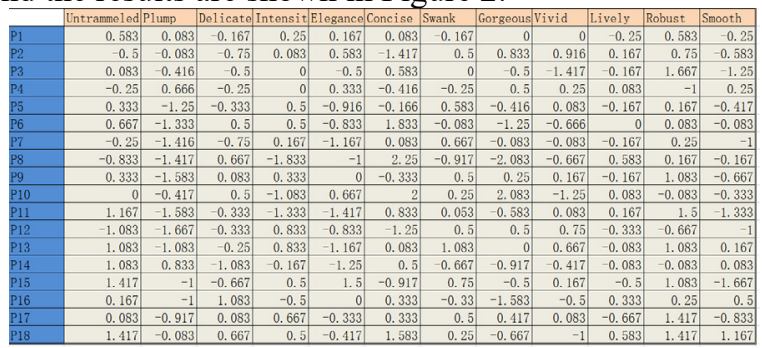

Figure 2. Expert scoring weighted average value table.

Weighted average value of expert's score is input into SPSS software for factor analysis. The main objective of the factor analysis method is to reduce the number of variables and use the factor analysis to obtain contribution values of the eigenvalues and variance, which can explain the product's intentional vocabulary with the least factors ${ }^{[10]}$. From Table 3 , it can be seen that the decoration of Yaozhou Kiln of the Five Dynasties was greatly influenced by two principal components, which were from components 1 to component 5, and their initial eigenvalues were all bigger than 1 , and the variance contribution values were $34.226 \%, 15.415 \%, 13.441 \%$, and $10.262 \%, 9.597 \%$, respectively, which can get the variance of $82.941 \%$ of the original variable. The two mainly components can represent the information of all variables. So, SPSS selects the five principal components to extract the sum of squares and replaces all the original variables with these 5 principal components. As shown in the Table 3.

The concept of gravel chart comes from geology. There are often many small gravels below the rock slope [11]. The gravel chart has feature values as the vertical axis and components on the horizontal axis. The steep part of the chart has a large feature value, which contains much information. And has the flat part has a small feature value and contains little information. From Table 4 , it can be seen intuitively that component 1 contains most of the information. It starts at component 6 to changing to be flatter. From the gravel chart shown in Figure 4 , there are 5 principal components with eigenvalues $\geq 1$. Before the fifth inflection point, the eigenvalues are generally higher and are connected into a steep polyline. From the fifth inflection point, the linear gradient becomes gentle. The gravel chart shows that this data is suitable for applying factor analysis to reduce the dimension. It is appropriate to analyze these five factors. 
Table.3 Characteristic Root and Variance Contribution.

\begin{tabular}{llll}
\hline No & $\begin{array}{c}\text { contribution values of the } \\
\text { eigenvalues }\end{array}$ & $\begin{array}{c}\text { contribution values of the } \\
\text { variance } \%\end{array}$ & accumulation\% \\
\hline 1 & 4.107 & 34.226 & 34.226 \\
2 & 1.850 & 15.415 & 49.641 \\
3 & 1.613 & 13.441 & 63.082 \\
4 & 1.231 & 10.262 & 73.343 \\
5 & 1.152 & 9.597 & 82.940 \\
6 & 0.717 & 5.972 & 88.912 \\
7 & 0.623 & 5.193 & 94.105 \\
8 & 0.362 & 3.015 & 97.121 \\
9 & 0.223 & 1.855 & 98.976 \\
10 & 0.070 & 0.584 & 99.560 \\
11 & 0.042 & 0.353 & 99.913 \\
12 & 0.010 & 0.087 & 100.000 \\
\hline
\end{tabular}

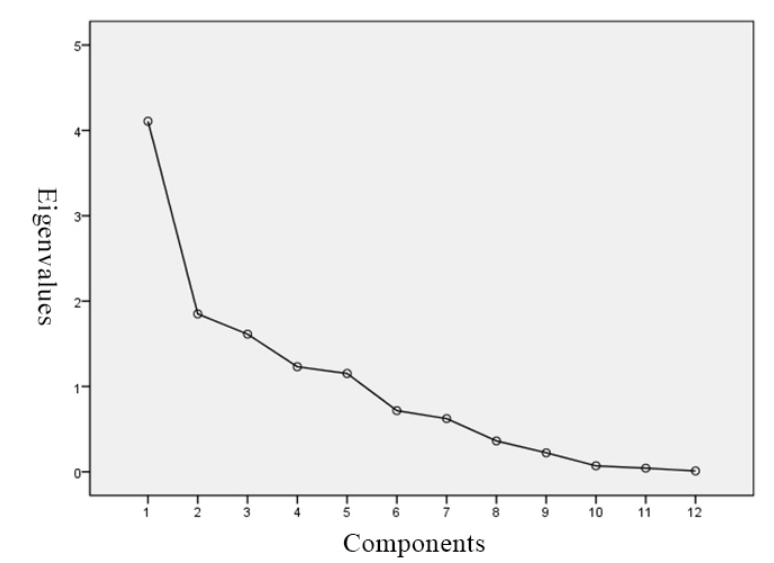

Figure 4. Gravel char.

The composition matrix is derived from the mainly component extraction method. From the Table 4, it can be seen that emotional adjectives are most affected by component 1 , and component 1 plays an important role in the overall data. Therefore, the data of component 1 is mainly referenced. And according to the relevant values of component 1 we can draw a conclusion that concise, lively and delicate are the 3 most important factors of expert statistics. Among them, the most consummate and valued factor elected by the consumers are the concise, then the liveliness ranks second, and the last is fresh. The experts pay less attention to delicate and vividness. According to the statistical analysis results, finally, we chosen the concise, then the liveliness ranks second, and the last is fresh as a Five Dynasties Yaozhou Kiln utensils decoration design factor. the concise, then the liveliness ranks second, and the last is fresh.
Table 3. Component Matrix.

\begin{tabular}{cccccc}
\hline Types & 1 & 2 & 3 & 4 & 5 \\
\hline Untrammeled & 0.031 & 0.572 & 0.680 & -0.196 & -0.114 \\
Plump & 0.125 & -0.556 & 0.696 & -0.152 & -0.361 \\
Delicate & 0.672 & 0.073 & -0.019 & 0.316 & 0.605 \\
Intensity & -0.667 & 0.086 & 0.337 & -0.320 & 0.387 \\
Elegance & -0.264 & -0.344 & 0.432 & 0.617 & 0.043 \\
Concise & 0.848 & 0.262 & 0.031 & 0.200 & -0.004 \\
Swank & -0.772 & 0.312 & 0.097 & 0.135 & 0.408 \\
Gorgeous & -0.542 & -0.390 & 0.178 & 0.477 & 0.112 \\
Vivid & -0.713 & -0.182 & -0.220 & -0.356 & 0.202 \\
Lively & 0.801 & -0.125 & 0.007 & -0.079 & 0.173 \\
Robust & -0.107 & 0.797 & 0.293 & 0.201 & -0.110 \\
Smooth & 0.594 & -0.282 & 0.434 & -0.358 & 0.479 \\
\hline & & & & & \\
\hline
\end{tabular}

\subsection{Creation design of the Five-Dynasties Yaozhou porcelain lotus flower ornament}

During the period of the Five Dynasties, porcelain in Yaozhou Kiln was mainly celadon firing, and utensil types were tableware, wine sets, and tea sets. And it is often made on a piece of artifact in the form of a sculpture, which pays great attention to the artistic effect of three-dimensional space and plays a prominent role in the vividness of the modeling of objects. The tea and water separation cup are used as the main body of the Yaozhou Kiln furniture design. As shown in Figure 5, there is a pot consisting of a cup, a lid and three parts, which can satisfy the use of brewing tea and tea alone, and it is easy to store and use. The design is functionally portable and beautiful, simplifies the traditional tea drinking process, which is concise, lively, and become the main key factor of design factors. The cup mouth adopts the lotus flower mouth design and pays tribute to the beauty of the traditional Yaozhou Kiln culture. Lotus flower design extraction factor of the Five-Dynasties Yaozhou Kiln are used on the body of the cup. So, the body's simple lotus style segmentation is both elegance and delicate. The lid with a lively design factor use a rotating design makes this product suitable for movement, changing the unfit situation of traditional tea set and modern home or office environment. 


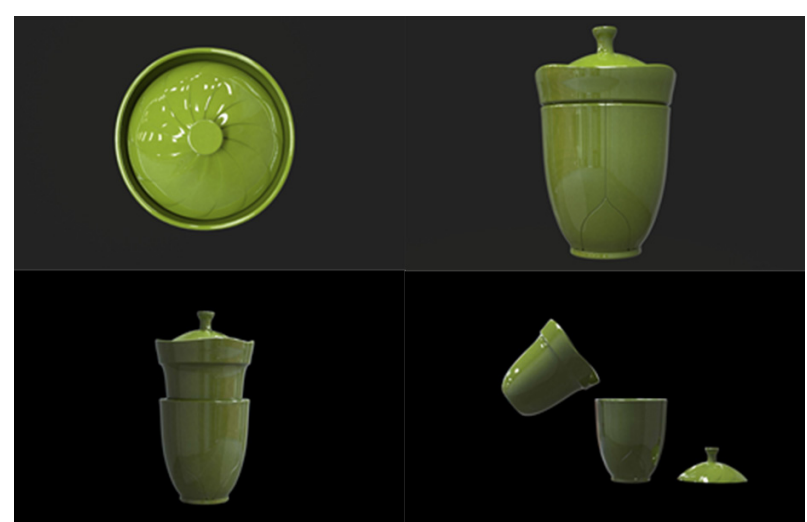

Figure 5. Creation Design of Lotus Flower Ornaments in Yaozhou Kiln in the Five Dynasties.

\section{Conclusion}

Yaozhou Kiln has rich cultural connotations and different levels of research in various fields. At present, the research of Yaozhou Kiln arts tends that were lied more stress on the past than on the present, making its product system tend to be conservative and self-styled. Tradition and modernity are not binary oppositions, but interconnected, expressed, and advanced. The idealized Yaozhou Kiln cultural and creative product not only needs the characteristics of inheriting the traditional forms, but also demands the artistic appeal of the current culture. They need to inherit the semantics of the traditional Yaozhou Kiln and break through the traditional design logic and shaping of the Yaozhou Kiln. Seeking the symbolic significance and new aesthetic value of ceramic art in the new era is to find a new opportunity for the development of Yaozhou Kiln. Therefore, effective design production is needed to penetrate into the people's current life. This article extracts the design elements of the important lotus ornament in the development of the Yaozhou Kiln culture by using the semantic difference method as a new design method, which can more efficiently and conveniently protect and disseminate precious Yao porcelain ornamentation. Through the analysis of the typical characteristics of the ceramic decoration of the Yaozhou Kiln in the Five Dynasties and the influence factors of the composition of the adjectives, the linear and recessive features were extracted, which proved that the semantic difference method is an effective method for extracting traditional cultural elements.

\section{Acknowledgements}

This study was supported by General project of Shanxi Art Humanities and Social Science Foundation (Grant Number sy2016048); General project of the humanities and social sciences fund of the Shanxi Provincial Education Department (Grant Number 17JK0524) ; $\mathrm{Xi}$ 'an science and technology university science research project humanities general project (Grant Number 106256091703).

\section{References}

1. Gou Bingchen, $\mathrm{Yu}$ Hui,Li Zhenfang,et al.Application of culture gene extraction and design in Banpo painted pottery[J].Journal of Northwestern Polytechnical University(Social Sciences),2011, 31(4): 66-69, 104.

2. Wang Weiwei,Liu Yin,Wang Yi. Extraction of Design Factors of Saline Soil Culture Based on Visual Correlation Analysis[J].Journal of Graphics.2016.37(1):60-65

3. Zhou Xiaozhou,Xue Chengqi,et al. Extraction of heritable factors of product family design DNA [J]. Journal of Southeast University(Natural Science Edition), 2016.46 (6) :1192-1196

4. JAY P M. Speaking the Buick Language: Capturing, Under-standing and Exploring Brand Identity with Shape Gram-mars[J]. Design Studies,2004.25(1): 19.

5. RAPHAELLE D, JEROME P, XAVIER F, et al. Identi-fication of Sensory Variables Towards the Integration of User Requirements into Preliminary Design[J]. In-ternational Journal of Industrial Ergonomics, 2007(37): 1-1.

6. Dou Jinhua. Design of walking aids for disabled persons based on semantic differential method [J]. Journal of Machine Design, 2014.31(6):118-121

7. Xu Jiang,Wang Haixian,Sun Shouqian.Method of product generative design based on style evolution model [J]. Journal of Southeast University(Natural Science Edition),.2012.42(4):654-657

8. Liu Zijian,Liang Jinfeng. Yaozhou Kiln Ceramic Decoration Based on Product Design DNA [J]. Packaging Engineering.2016.37(4):45-51

9. Zhao Chunyan. Folk Customs Artistic Style of Ornament's Veins on Yao Zhou Kiln Pottery Art [D]. Xi'an:Xi'an Academy of Fine Arts,2008.

10. Xu Zhanmin,Li Yang. Application of Extraction Model of Product Design Factors of Floral Culture [J]. Journal of Graphics.2017.38(1):45-51

11. Lu Zhaolin,Li Shengbo,Zhang Yue, et al.Product design oriented to industrial design.DNA Research status and progress [J]. Journal of Machine Design,2014.31(12):432-436. 